\title{
RASSF10 is Epigenetically Inactivated and Suppresses Cell Proliferation and Induces Cell Apoptosis by Activating the p53 Signalling Pathway in Papillary Thyroid Carcinoma Cancer
}

\author{
Cheng Fan ${ }^{\mathrm{a}}$ Wendy Wang ${ }^{\mathrm{b}}$ Ji Jin ${ }^{\mathrm{b}}$ Zhuo Yu ${ }^{\mathrm{b}} \quad$ Xiping Xin \\ aDepartment of Geriatrics, Institute of Geriatrics, Union Hospital, Tongji Medical College, Huazhong \\ University of Science and Technology, Wuhan, China; bepartments of Anatomy and Cell Biology, \\ Schulich School of Medicine and Dentistry, University of Western Ontario, London, Ontario, Canada
}

\section{Key Words}

RASSF10 • p53 Signalling Pathway • Apoptosis • Papillary Thyroid Carcinoma

\begin{abstract}
Objectives: We aimed to confirm whether RASSF10 activated the p53 signalling pathway, thereby modulating cell proliferation, migration, invasion, and apoptosis in papillary thyroid carcinoma (PTC) cells. Methods: A total of 108 PTC tissue samples and normal adjacent tissues were obtained. RT-PCR and Western blotting analyses were performed to detect RASSF10 expression, and methylation levels of RASSF10 were estimated by methylation-specific PCR (MSP). We also detected the expression and methylation status of RASSF10 in both a human PTC cell line (K1) and a normal thyroid cell line (FRTL5). After transfection of cells with empty vector pcDNA3.1, pcDNA3.1-RASSF10, p53 siRNA and shRASSF10, Coulter counter, colonyformation, wound healing, Transwell and flow cytometry analyses were performed to examine the role of RASSF10 in cell proliferation, migration, invasion, and apoptosis. Finally, the expression of p53, p21, Bcl-2 and Bax were detected using Western Blotting analyses. Results: RASSF10 expression in PTC tissues was significantly lower and hyper-methylated compared to normal adjacent tissues. In addition, RASSF10 was significantly down-regulated and hypermethylated in K1 cells compared to FRTL5 cells. In addition, suppressed proliferation and significantly induced apoptosis of K1 cells were observed after transfection with pcDNA3.1RASSF10 $(P<0.05)$. Furthermore, RASSF10 activated the p53 signalling pathway and regulated the expression of p53, p21, Bcl-2 and Bax. Furthermore, p53 siRNA could antagonize the effects of RASSF10 in K1 cells. Conclusions: RASSF10 induces apoptosis in PTC cells by activating the p53 signalling pathway, indicating its role as a treatment target for PTC.
\end{abstract}




\section{Introduction}

As one of the most prevailing endocrine malignancies, the incidence of thyroid cancer (TC) has been steadily increasing, with an incidence of 6.10/100,000 in females and 1.90/100,000 in males [1,2]. According to a statistical review, 229,900 new female TC cases and 70,000 new male TC cases were documented in 2012 [1,2]. Papillary thyroid carcinoma (PTC) is the most frequent subtype of TC, accounting for approximately $75-85 \%$ of TC cases $[2,3]$. PTC patients encountering adjacent tissue invasion or lymphatic metastasis are prone to suffer from unfavourable prognosis $[4,5]$. Although PTC development has been hypothesized to be induced by various factors of heredity, hormone secretion and environmental hazards, the inherent PTC pathogenesis remains ambiguous, particularly the mechanism underlying the regulation of the invasion and migration of PTC [6].

The RAS association domain family (RASSF) proteins are correlated with Ras-like small guanosine triphosphate (GTP)-binding proteins and are involved in a series of cellular processes, including cell growth migration, adhesion and apoptosis [7]. They consist of 10 members (RASSF1- RASSF10) and are divided into classical RASSF (i.e., RASSF1- RASSF6) and N-terminal RASSF (i.e., RASSF7-RASSF10) [8]. Classical RASSF proteins have a featured protein-protein interaction domain called SARAH (SARAH: SAlvador, RAssf, Hippo) in the C-terminus, while N-terminal RASSF proteins (e.g., RASSF10) are identifiable due to their SARAH domain [9]. RASSF10, which is located on chromosome 11p15.2 near the RRAS2 oncogene, contains a $\mathrm{CpG}$ island in the promoter region that is responsible for methylation and can contribute to tumourigenesis $[8,10]$. Epigenetically inactivated RASSF10 has been suggested in diverse tumours, including prostate carcinoma, glioblastoma, skin malignant melanoma and thyroid cancer [11-14]. Previous findings have indicated that RASSF10 might suppress the growth of colorectal and hepatocellular carcinomas, as well as stimulate cell cycle arrest and cell apoptosis by activating p53 signalling [15-18]. The dominant components of p53 signalling, including p53, p21, Bcl-2 and Bax, have been extensively studied in carcinomas $[16,19]$. Among these factors, the p53 mutation appears to be the most frequent genetic alteration observed in tumour cells. For instance, the mutated form of p53 regularly appears in undifferentiated thyroid carcinomas [20-22], and MDM2, another pivotal element of p53 signalling, can directly catalyse p53 degradation by exacerbating p53 ubiquitination $[18,23]$.

However, how RASSF10 and p53 signalling are coordinated and induce the formation and progression of PTC remain elusive, although RASSF10 and p53 have been suggested to be independent correlated with TC. Thus, the present study was designed to illustrate the epigenetic deactivation of RASSF10 in PTC and the role of p53 signalling in RASSF10 through which PTC cell apoptosis is epigenetically regulated.

\section{Materials and Methods}

\section{Subjects}

A total of 108 PTC tissue samples and normal adjacent tissues were obtained from Union Hospital, Tongji Medical College, Huazhong University of Science and Technology, between January 2011 and January 2014. Clinical and pathological data, including gender, age, tumour size, pathological types, lymph node metastasis and histological grade were recorded. Tissues were promptly frozen in liquid nitrogen after thyroidectomy and stored at $-80^{\circ} \mathrm{C}$ until further use. All samples were diagnosed by histological review. None of these patients received preoperative chemotherapy or radiotherapy. The study was approved by the ethics committee of Union Hospital, Tongji Medical College, Huazhong University of Science and Technology, and all participants provided informed consent.

The human PTC cell line (K1) and the normal thyroid cell line (FRTL5) were acquired from the Chinese Academy of Sciences (Shanghai, China). Both K1 and FRTL5 cells were cultured in RPMI 1640 medium containing $10 \%$ newborn bovine serum, $100 \mathrm{U} / \mathrm{mL}$ penicillin and $100 \mathrm{U} / \mathrm{mL}$ streptomycin sulfate in a 5\% $\mathrm{CO}_{2}$ atmosphere at $37^{\circ} \mathrm{C}$.

\section{KARGER}


RNA extraction and RT-PCR

RNA was isolated from tissue and cell samples using Trizol reagent (Invitrogen, Carlsbad, CA, USA) according to the manufacturer's instructions. Next, RNA was reversed transcribed into cDNA using SuperScript III (Invitrogen) and used as the template for real time-PCR (RT-PCR) assays. The conditions of RT-PCR were performed as previously described [24]. The sequences of the primers used in this experiment are listed in Table 1. The relative expression of RASSF10 was normalized to $\beta$-actin.

Methylation-specific PCR (MSP) and bisulfite genomic sequencing (BGS)

Genomic DNA was isolated from samples using QIAamp DNA Mini kit (Qiagen, Hilden, Germany). Bisulfite modification of DNA and MSP were performed as described by Li et al. [25]. Bisulfite-modified DNA was amplified using methylation-specific PCR primers listed in Table 1. The methylation levels in cells were estimated by cloning PCR products amplified from bisulfite-treated DNA using BGS primers as previously described [26].

\section{Demethylation treatment}

K1 cells were treated with $2 \mu \mathrm{M}$ 5-aza-2'-deoxycytidine (5-aza-dC, Sigma, USA), a DNA methylation transferase inhibitor for 4 days to achieve demethylation. Untreated K1 cells were regarded as negative controls.

\section{Western Blotting analyses}

Proteins were extracted from the cells using the CytoBuster Protein Extraction Reagent (Merck Chemicals, UK), and the protein concentrations were determined using a BCA assay kit (Beyotime). Following protein separation by SDS-polyacrylamide gel electrophoresis (PAGE), proteins were transferred onto equilibrated polyvinylidene difluoride membranes (Jianglai Biotechnology, China). After incubation with specific primary antibodies, including rabbit anti-human RASSF10 (Abcam, ab113105), p53 (Santa Cruz, sc-6243), p21 (Santa Cruz, sc-6246), Bcl-2 (Santa Cruz, sc-7382), Bax (Santa Cruz, sc-526), E-cadherin (BD biosciences, 610181), N-cadherin (Santa Cruz, 8C11), Vimentin, ZO-1 (Santa Cruz, sc-6260), TCF-8/ ZEB1 (Cell Signaling Technology, 3396) and $\beta$-catenin (Cell Signaling Technology, 9582) at $4^{\circ} \mathrm{C}$ overnight and incubation with secondary antibodies, including horseradish peroxidase (HRP) conjugated goat anti-rabbit IgG (Santa Cruz, sc-2004), proteins were visualized using ECL Plus Western Blotting Detection Reagents (GE Healthcare, UK). $\beta$-actin served as the internal reference.

\section{Expression vector construction and transfection}

The open reading frame (ORF) of the RASSF10 gene was generated by RT-PCR, and p53 siRNA and shRASSF10 were purchased from OriGene Technologies (Rockville, USA). Next, RASSF10, p53 siRNA and shRASSF10 were cloned into the pcDNA3.1 expression vector according to the manufacturer's guidelines (Invitrogen). The constructed sequence was further confirmed by sequencing. Expression vectors were transfected into K1 cells with low RASSF10 expression using Lipofectamine 2000 (Invitrogen). Next, the cells were divided into five groups: control group (cells were transfected with empty vector pcDNA3.1), RASSF10 group (cells were transfected with pcDNA3.1-RASSF10), RASSF10 + p53 siRNA group (cells were transfected with pcDNA3.1-RASSF10 and p53 siRNA), shcontrol group (cells were transfected with shcontrol), and shRASSF10 group (cells were transfected with shRASSF10). Stably transfected cells, which had been cultured for more than 12 days, were selected using G418 (Roche, USA).

Table 1. Primer sequences for RT-PCR and methylationspecific PCR. For, forward; rev, reverse; $\mathrm{M}$, methylation; $\mathrm{U}$, unmethylation; B, bisulfite genomic sequencing

\begin{tabular}{llll}
\hline Gene & \multicolumn{2}{l}{ Primer } & Size (bp) \\
\hline \multirow{2}{*}{ RASSF10 } & for & 5'-CCATGACCCAGGAGAAACAG-3' & \multirow{2}{*}{26} \\
& rev & 5'-TGCTGGCGAATTGTGTGGTC-3' & \\
RASSF10-M & for & 5'-GGGTATTTTGGGTAGAGTTAGAGC-3' & \\
& rev & 5'-AAACAAACTAAAAAACGACTACGAC-3' & 126 \\
RASSF10-U & for & 5'-GGGTATTTTGGGTAGAGTTAGAGTG-3' & \\
& rev & 5'-AAACAAACTAAAAAACAACTACAAC-3' & 126 \\
\multirow{2}{*}{ RASSF10-B } & for & 5'-TTTGGGTTTTGAGTTTTTTGTATTT-3' & \multirow{2}{*}{257} \\
& rev & 5'-ACTACACTAACCCTATTCCCCC-3' & \\
\multirow{2}{*}{ 3-actin } & for & 5'-TCGACAACGGCTCCGGCAT-3' & \multirow{2}{*}{241} \\
& rev & 5'-AAGGTGTGGTGCCAGATTTTC-3' & \\
\hline
\end{tabular}


Cell proliferation and apoptosis assay

Cells $\left(5 \times 10^{3}\right.$ cells/well) were seeded onto 24 -well plates and cultured for 1-4 days. Next, the cells were harvested and quantified using Coulter counter (Beckman Coulter, Fullerton, CA, USA). For apoptosis analysis, cells $\left(2.5 \times 10^{5}\right.$ cells/well $)$ were seeded onto 6 -well plates without the addition of serum for 1 day, and culture medium containing $10 \%$ FBS was used to replace the previous culture medium. Apoptosis was detected using the Annexin V-FITC/PI Apoptosis Detection Kit (BD Biosciences, USA) under FACScan Flow Cytometer 24 hours after medium replacement. All assays were performed in triplicate.

Colony-formation assay

Cells $\left(1 \times 10^{3}\right)$ were plated onto a $10-\mathrm{cm}$ dish for 2 weeks. The cells were then washed twice with phosphate buffered saline (PBS), fixed with acetic acid/methanol (1:3, v/v), and stained with crystal violet $(0.5 \%)$. The colony number was quantified under a microscope, and the assay was performed in triplicate.

Wound healing assay

Each well contained approximately $2 \times 10^{5}$ cells. When the cell density reached $80 \%$, a line was scratched vertically in the middle of the plates using a pipette tip. After three washes with PBS, the plates were incubated in growth medium. The wound widths were measured under a microscope after $0 \mathrm{hrs}, 24$ hrs and 48 hrs.

Transwell assay

Transwell chambers were prepared according to the manufacturer's instructions, and the cell density was adjusted to prepare the cell suspension solution. In addition, transwell chambers were incubated for $12 \mathrm{~h}$ after inoculation, and the medium was removed and washed gently with PBS three times. The corresponding data during these procedures were recorded.

\section{Statistical analysis}

Data obtained from the three independent experiments are expressed as the mean \pm standard deviation (SD). Any difference in variables between the PTC and adjacent normal tissues was analysed using the paired t-test. Moreover, Student's t-test and one-way analysis of variance (ANOVA) was performed to compare the difference between different groups of cells. All data were analysed using SPSS 21.0 software. $P<0.05$ was considered statistically significant.

\section{Results}

\section{Subject characteristics}

The clinical characteristics of 108 case subjects are shown in Table 2. The average age of the patients was $50.86 \pm 11.85$ years old, and the proportion of males was significantly less than that of females $(P<0.05)$. As suggested by the World Health Organization (WHO) histological classification [27], 94 PTC patients were diagnosed with cystic papillary thyroid carcinoma (cPTC), and 14 patients were diagnosed with other types of PTC. There were 17 cases with extrathyroidal extension and 91 cases without extrathyroidal extension. For tumour size, 78 and 24 patients had tumours with the diameters of $<2 \mathrm{~cm}, 2-4 \mathrm{~cm}$, respectively, and the remaining 6 patients had tumours with diameters $>4 \mathrm{~cm}$.

\section{RASSF10 was constantly methylated in PTC tissues}

We used MSP to determine whether methylation of RASSF10 occurred in PTC cancer. The results showed that RASSF10 was methylated in $63.9 .0 \%(69 / 108)$ of PTC samples, while only $8.3 \%$ (9/108) of cases were methylated in adjacent tissues (Fig. 1A, Table 2). Moreover, RASSF10 methylation was notably associated with tumour size $(P<0.05)$, although no significant association was discovered with age, sex, histological variant, extrathyroidal extension and distant metastasis $(P>0.05$, Table 2$)$. Furthermore, the expression of RASSF10 was detected using Western blotting analyses in PTC tissues and paired normal adjacent 
Table 2 Clinicopathological features of the study cohort and correlation of RASSF10 methylation to clinicopathological data of 108 PTC

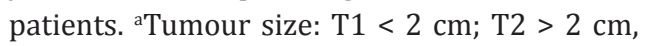
but $\leqq 4 \mathrm{~cm}$; T3 $>4 \mathrm{~cm} .{ }^{*} p$ values were obtained from the chi-square test, $P<0.05$ represents a significant difference

\begin{tabular}{lcccc}
\hline Characteristics & \multicolumn{4}{c}{$\begin{array}{c}\text { RASSF10 methylation status } \\
\text { methylated } \\
\text { unmethylated } P \text {-value }\end{array}$} \\
\hline $\begin{array}{l}\text { Age } \\
\leqq 50\end{array}$ & 41 & 27 & 14 & 0.7395 \\
$>50$ & 67 & 42 & 25 & \\
Sex & & & & \\
male & 27 & 18 & 9 & 0.7286 \\
female & 81 & 51 & 30 & \\
Histological variant & & & & \\
cPTC & 94 & 60 & 34 & 0.9736 \\
other & 14 & 9 & 5 & \\
Tumour size & & & & \\
T1 & 78 & 44 & 34 & $0.0333 *$ \\
T2 & 24 & 20 & 4 & \\
T3 & 6 & 5 & 1 & \\
Extrathyroidal extension & & & & \\
yes & 17 & 9 & 8 & 0.3059 \\
no & 91 & 60 & 31 & \\
Distant metastasis & & & & \\
yes & 0 & 0 & 0 & 1 \\
no & 108 & 69 & 39 & \\
\hline
\end{tabular}

Fig. 1. Results of RASSF10 methylation and expression in PTC. (A) Methylation rate of RASSF10 in PTC tissue samples and adjacent tissue samples. PTC: papillary thyroid carcinoma. (B-C) Relative RASSF10 expression in 50 PTC tissue samples normalized to adjacent tissue samples as detected by Western blotting analyses.

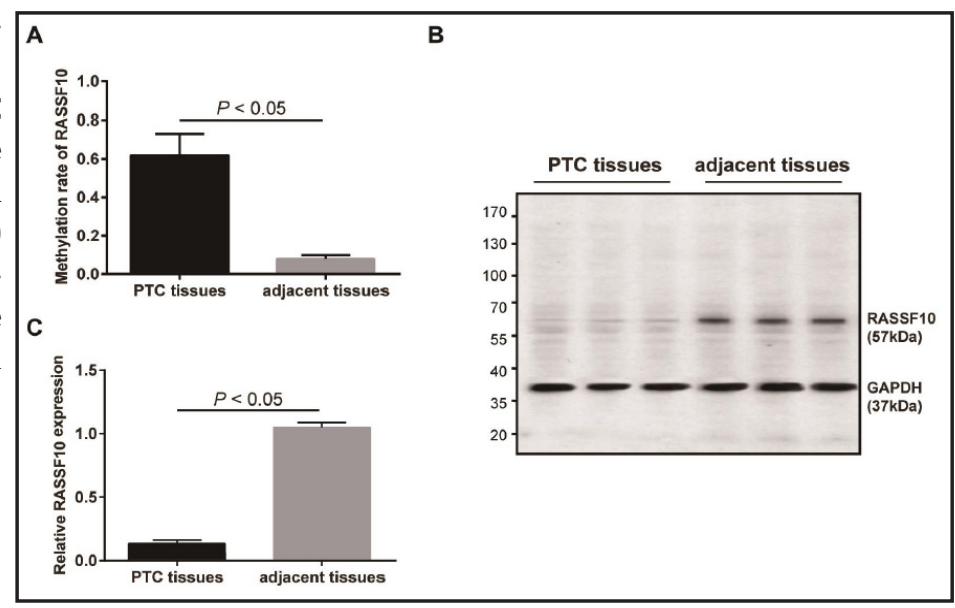

Fig. 2. Expression and methylation status of RASSF10 in PTC cells. (A) RASSF10 was frequently silenced and methylated in the PTC cell line $\mathrm{K} 1$, but was expressed and unmethylated in the normal thyroid cell line FRTL5. (B) Pharmacological demethylation with $2 \mu \mathrm{M} 5$-azadC restored RASSF10 expression. (C) Bisulfite sequencing results of RASSF10 in K1 and FRTL5 cells. $\beta$-actin: internal control.

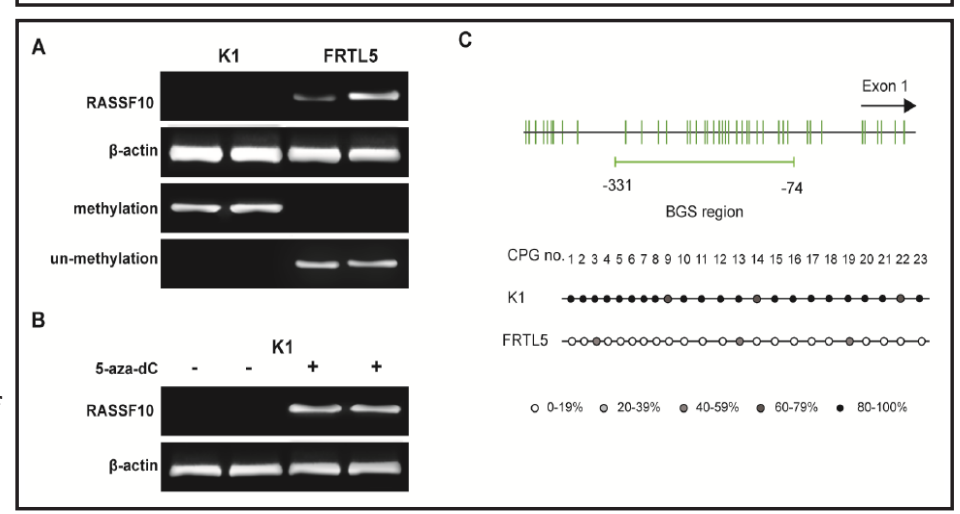

tissues. These results showed that RASSF10 expression was significantly down-regulated in PTC tissues compared to adjacent tissues (Fig. 1B-C).

RASSF10 expression was down-regulated by promoter hyper-methylation in PTC cells

To determine RASSF10 expression and methylation, RT-PCR and MSP was employed in human PTC cells (K1) and normal thyroid cells (FRTL5). As shown in Fig. 2A, loss of RASSF10 expression was detected in K1 cells, whereas RASSF10 was highly expressed in FRTL5 cells. Moreover, complete methylation was detected in K1 cells, while non-methylation was observed in FRTL5 cells. To investigate whether RASSF10 expression was regulated by promoter hyper-methylation, the PTC cell line K1 was treated with 5-aza-dC. After the 
Fig. 3. RASSF10 regulated cell proliferation, migration and invasion by p53 regulation. (A) The expression of RASSF10 and p53 were detected by RT-PCR and western blotting analyses. (B-C) Growth curves and colony formation results represent the effects of RASSF10 and p53 on cell growth in $\mathrm{K} 1$ (B) and FRTL5 (C). (D-E) The results of the wound healing assay showed that RASSF10 and p53 regulated cell migration in $\mathrm{K} 1$ (D) and FRTL5 (E). (F-G) Transwell assay showed the effects of RASSF and p53 on cell invasion in K1 (F) and FRTL5 (F) ${ }^{*} P<0.05$ versus control group, ${ }^{\#} P<0.05$ versus the RASSF10 group.

application of treatments with 5-aza-dC, RASSF10 expression was reexpressed in $\mathrm{K} 1$ cells (Fig. 2B), suggesting that RASSF10 promoter methylation may repress its transcription. In addition, BGS was performed to further confirm the methylation efficiency of the RASSF10 promoter in $\mathrm{K} 1$ and FRTL5 cells, and its results were consistent with those obtained from MSP (Fig. 2C). Taken together, these results indicated that RASSF10 expression is downregulated by promoter hyper-methylation in PTC cancer.

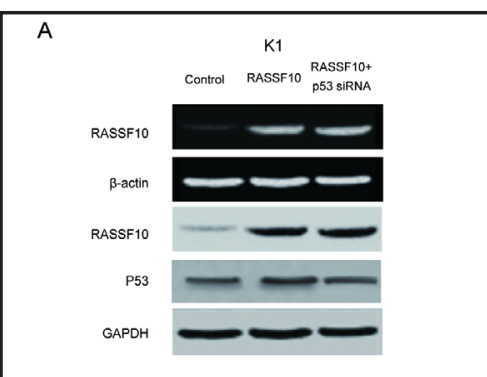

B
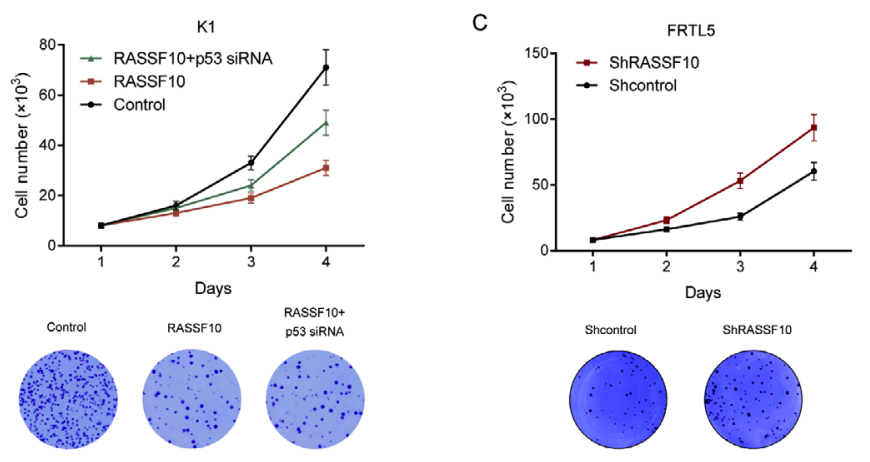

D

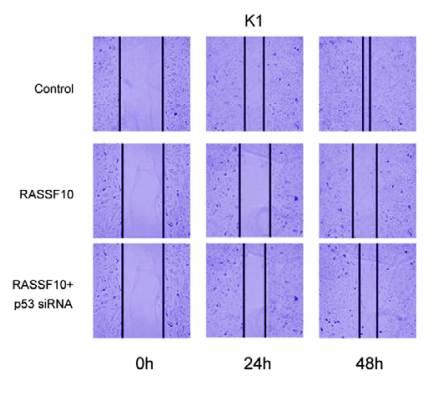

E

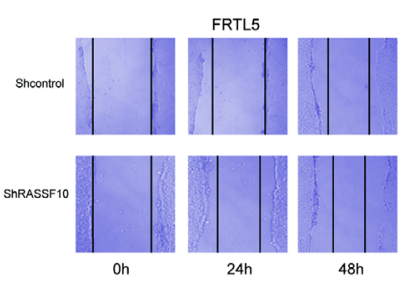

F

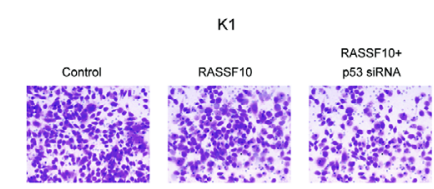

G

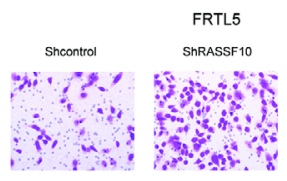

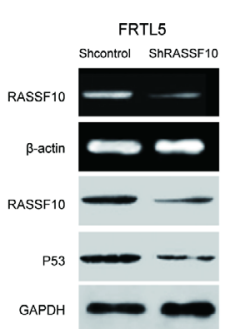
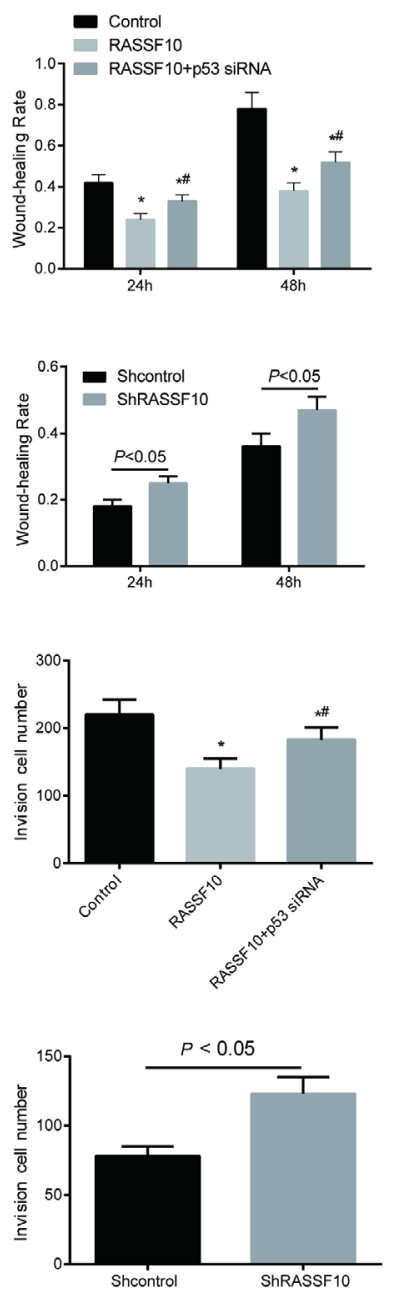
Fig. 4. The expression of E-cadherin, $\mathrm{N}$ cadherin, Vimentin, ZO-1, TCF-8/ZEB-1 and $\beta$-catenin was detected in $\mathrm{K} 1$ (A) and FRTL5 (B) cells by western blotting analyses. $\beta$-actin: internal control.

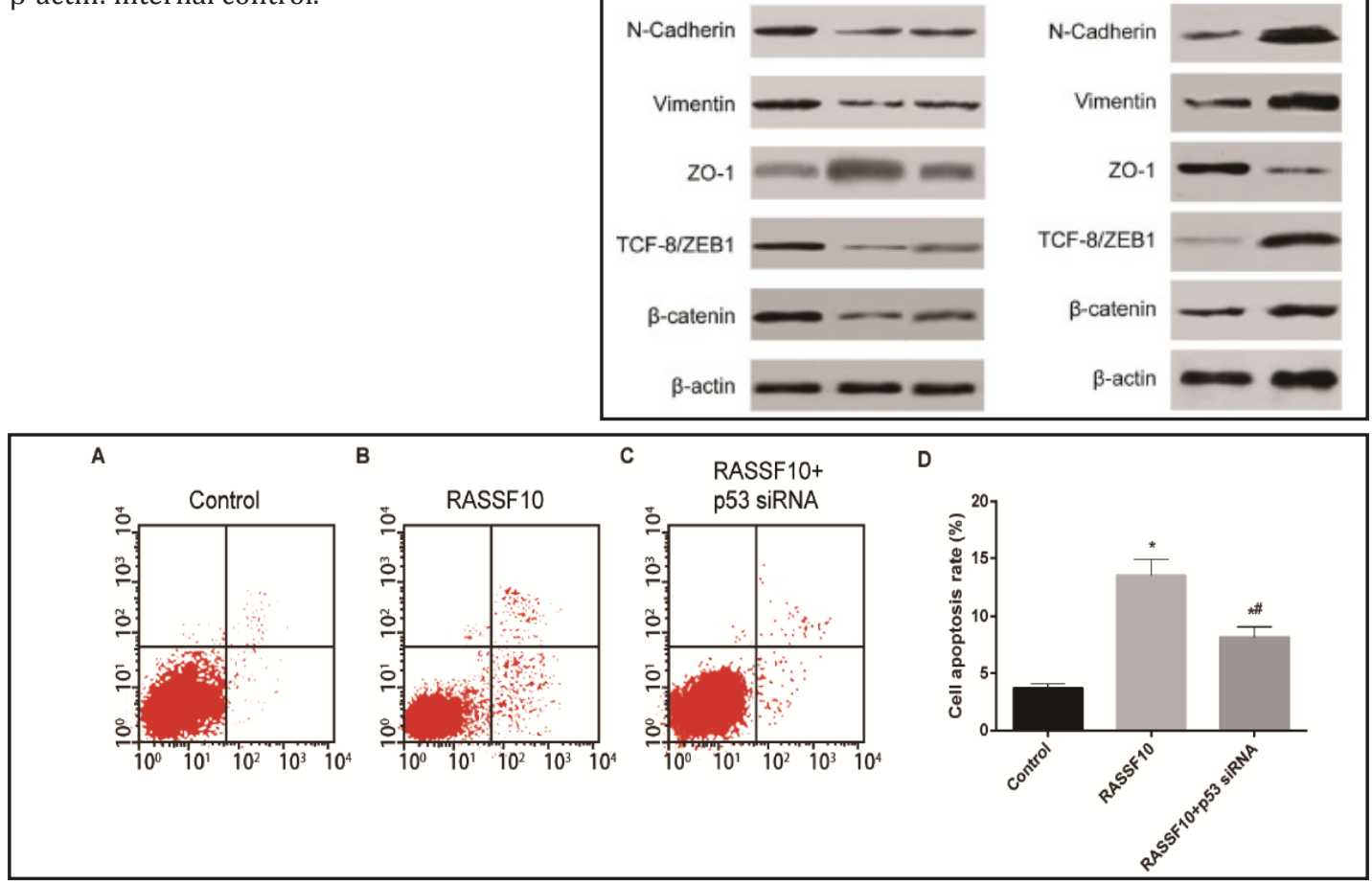

Fig. 5. RASSF10 and p53 regulated PTC cell apoptosis. Apoptosis was determined using flow cytometry analysis of Annexin V/PI double-stained K1 cells. ${ }^{*} P<0.05$ versus control group, ${ }^{\#} P<0.05$ versus RASSF10 group.

RASSF10 inhibited cell proliferation, migration, invasion and induced apoptosis in PTC cells by activating the 553 signalling pathway

RT-PCR and Western blotting assays revealed that the up-regulation of RASSF10 mRNA and protein were achieved in K1 cells via transfection of pcDNA3.1-RASSF10. Transfection of shRASSF10 can down-regulate RASSF10 mRNA and protein expression. In addition, transfection of pcDNA3.1-RASSF10 could also up-regulate p53 expression, whereas shRASSF10 could decrease the expression of p53 protein (Fig. 3A). The effects of RASSF10 or p53 siRNA on cell proliferation, migration, invasion, and apoptosis were analysed using a Coulter counter and colony-formation, wound healing, Transwell and flow cytometry assays. As shown in Fig. 3B-G, RASSF10 significantly attenuated K1 cell proliferation, migration and invasion in contrast to the pcDNA3.1-NC cells $(P<0.05)$, while pcDNA3.1-RASSF10 + p53 siRNA co-transfected cells remarkably accelerated cell proliferation, migration and invasion. These findings differed to cells that were only treated with pcDNA3.1-RASSF10 $(P<0.05)$. Furthermore, transfection of shRASSF10 could promote the proliferation, migration and invasion of FRTL5 cells $(P<0.05)$. These results indicated that RASSF10 inhibited the proliferation, migration and invasion of cells, but p53 siRNA significantly reversed the inhibitory effects of RASSF10 in cells. In addition, compared with the control group, RASSF10 group showed higher expression of E-cadherin and ZO-1 and lower expression of $\mathrm{N}$-cadherin, Vimentin, TCF-8/ZEB1 and $\beta$-catenin. Nevertheless, transfection of p53 siRNA or shRASSF10 contributed to opposing effects, demonstrating that RASSF10 can regulate epithelial-mesenchymal transition (EMT) by regulating p53 (Fig. 4).

Next, we measured apoptotic cells following up-regulation of RASSF10 or inhibition of p53 in K1 cells. As shown in Fig. 5, RASSF10 significantly induced apoptosis in K1 cells, 
while p53 siRNA significantly reversed the promoting effects, and pcDNA3.1-RASSF10 + p53 siRNA co-transfected cells had a remarkably lower apoptosis rate than the RASSF10 group $(P<0.05)$.

To investigate the role of RASSF 10 in the p53 signalling pathway, the expression levels of p21, Bcl-2 and Bax were detected using western blotting analyses. As shown in Fig. 6 , the expression of $\mathrm{p} 21$ was examined, and increased Bax and decreased Bcl-2 expression was observed following upregulation of RASSF10 expression in K1 cells. The effects of RASSF10 overexpression were antagonized by transfection of p53 siRNA or shRASSF10.

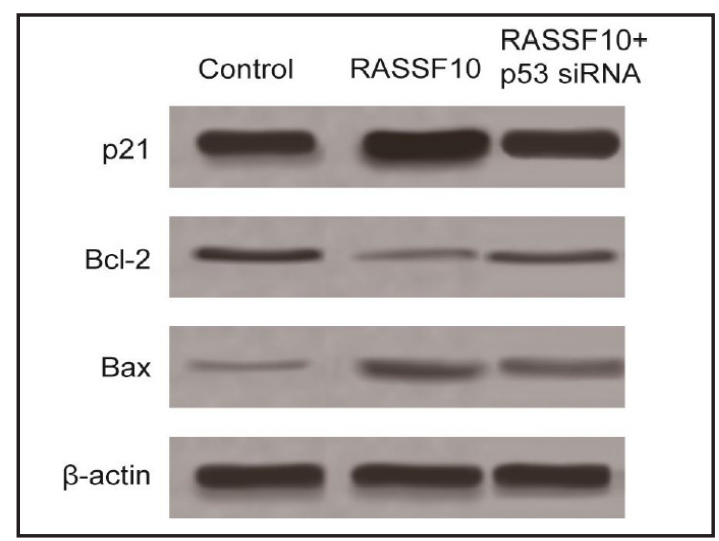

Fig. 6. The expression of P21, Bcl-2 and Bax in cells were examined by western blotting analyses.

Taken together, these results indicated that the p53 signalling pathway was activated by RASSF10 in PTC cells.

\section{Discussion}

The implication of dysregulated oncogenic expression in human PTC has been well reported in the current literature [28-30]. However, the potential association between tumour inhibitors of PTC and cellular processes has not been fully understood. Moreover, some PTC patients do not exhibit abnormal expression of some identified oncogenes [31]. Thus, we suspected unidentified genes and mechanisms might also exert crucial roles in thyroid tumourigenesis.

Epigenetic inactivation of tumour suppressor genes is an elementary event in the pathogenesis of malignant tumours, which is often associated with advanced or aggressive tumours, disease progression, and poor prognosis [32-34]. Several studies have indicated that at least eight of the RASSF members, including RASSF1 to RASSF10, are downregulated by promoter hypermethylation in diverse types of cancers [7, 8, 35-37]. RASSF proteins carry several characteristic domains and can function as adaptor proteins in several biological processes. Initial studies have demonstrated their interactions with diverse Ras GTPases and the Mammalian sterile 20-like kinases, as well as their tumour inhibitory role in cell apoptosis, the Hippo pathway, and cell cycle and cytoskeleton regulation [38]. Previous studies have reported the RASSF family as potential tumour suppressors and prognostic markers $[8,37]$. The current literature has also demonstrated that RASSF family members are critical to NF-kB and Wnt signalling pathways, which might be involved in epithelialmesenchymal transition (EMT) and cell motility [39, 40].

In this study, we indicated that the recently identified RASSF member RASSF10 gene is often silenced in PTC cells. Downregulation of RASSF10 is closely associated with aberrant promoter methylation. We found that pharmacological demethylation can restore the expression of RASSF10, suggesting that promoter methylation might overcome the inactivation of RASSF10 in PTC. As suggested by our data and data obtained in other studies, RASSF10 may encode a novel epigenetically inactivated candidate tumour inhibitor gene in thyroid tumourigenesis [41].

RASSF10 has been reported to function as a tumour suppressor in numerous cancers, including Merkel cell carcinoma, malignant melanoma, glioblastoma, pheochromocytomas, hepatocellular carcinoma, lung cancer, colorectal cancer, gastric cancer and prostate cancers $[11-13,16,26,42-45]$. To reveal the function and corresponding mechanism of RASSF10 in PTC, we investigated its effects on both carcinogenesis and tumour progression. Our results showed that overexpression of RASSF10 significantly attenuated cell proliferation, migration, and invasion and induced cell apoptosis in a human PTC cell line (K1), while p53 
siRNA significantly reversed the corresponding effects. Compared to the control group, the RASSF10 group exhibited higher expression of E-cadherin and ZO-1 and lower expression of N-cadherin, Vimentin, TCF-8/ZEB1 and $\beta$-catenin, while p53 siRNA contributed to the opposite effects. Thus, we suspected that RASSF10 could regulate epithelial-mesenchymal transition (EMT) by regulating p53. Taken together, these results further showed that the carcinogenesis and tumour progression in PTC cells were potentially affected by RASSF10 via activating the p53 signalling pathway. A similar study suggested that RASSF10 could inhibit the proliferation of colorectal cancer [16].

A previous study has reported that p53 encodes a transcription factor, which contributes to the transcriptional activation of a large number of targets, such as p21 and the proapoptotic protein Bax [46]. Thus, p53 is likely to affect upstream pro-apoptotic proteins to modulate their functions in the cytoplasm. We also found that overexpression of RASSF10 significantly increased the expression of p53, p21, and Bax, and decreased the expression of Bcl-2 in K1 cells, while p53 siRNA significantly decreased these corresponding effects. These results demonstrated that RASSF10 serves as a tumour inhibitor in PTC cells, and it has a potential role in carcinogenesis and tumour progression.

In summary, our study demonstrated that RASSF10 is a potential tumour inhibitor gene in PTC and is mainly inactivated by promoter methylation. RASSF10 contributes to the inhibition of carcinogenesis by facilitating cell apoptosis and suppressing cell proliferation, migration and invasion by activating the p53 signalling pathway. As a result, RASSF10 might act as a novel molecular target for the detection and treatment of PTC. However, further studies are needed to investigate the effect of RASSF10 on other cellular processes in PTC, such as cell adhesion and differentiation.

In summary, this study identified the tumour-suppressive role of RASSF10 in PTC, as assessed by RASSF10 facilitation of cell apoptosis via activating the p53 signalling pathway. However, further studies are needed to investigate the effect of RASSF10 on other cellular processes in PTC, such as cell adhesion and differentiation.

\section{Disclosure Statement}

The authors declare that they have no competing interests.

\section{References}

1 Torre LA, Bray F, Siegel RL, Ferlay J, Lortet-Tieulent J, Jemal A: Global cancer statistics, 2012. CA Cancer J Clin 2015;65:87-108.

2 La Vecchia C, Malvezzi M, Bosetti C, Garavello W, Bertuccio P, Levi F, Negri E: Thyroid cancer mortality and incidence: a global overview. Int J Cancer 2015;136:2187-2195.

3 Gong W, Yang S, Yang X, Guo F: Blood preoperative neutrophil-to-lymphocyte ratio is correlated with TNM stage in patients with papillary thyroid cancer. Clinics (Sao Paulo) 2016;71:311-314.

4 Wang Z, Zhang H, Zhang P, Dong W, He L: MicroRNA-663 suppresses cell invasion and migration by targeting transforming growth factor beta 1 in papillary thyroid carcinoma. Tumor Biol 2016;37:76337644.

5 Qu N, Zhang L, Wu WL, Ji QH, Lu ZW, Zhu YX, Lin DZ: Bilaterality weighs more than unilateral multifocality in predicting prognosis in papillary thyroid cancer. Tumour Biol 2016;37:8783-8789.

6 Pellegriti G, Frasca F, Regalbuto C, Squatrito S, Vigneri R: Worldwide increasing incidence of thyroid cancer: update on epidemiology and risk factors. J Cancer Epidemiol 2013;2013:965212.

7 Djos A, Martinsson T, Kogner P, Caren H: The RASSF gene family members RASSF5, RASSF6 and RASSF7 show frequent DNA methylation in neuroblastoma. Mol Cancer 2012;11:40.

8 Richter AM, Pfeifer GP, Dammann RH: The RASSF proteins in cancer; from epigenetic silencing to functional characterization. Biochim Biophys Acta 2009;1796:114-128. 


\section{Cellular Physiology Cell Physiol Biochem 2017;41:1229-1239 \begin{tabular}{l|l|l}
\hline and Biochemistry 10.1159/000464386 & $\begin{array}{l}\text { () 2017 The Author(s). Published by S. Karger AG, Basel } \\
\text { www.karger.com/cpb }\end{array}$
\end{tabular}

Fan et al.: RASSF10 Regulates p53 in PTC

- Kudo T, Ikeda M, Nishikawa M, Yang Z, Ohno K, Nakagawa K, Hata Y: The RASSF3 candidate tumor suppressor induces apoptosis and G1-S cell-cycle arrest via p53. Cancer Res 2012;72:2901-2911.

10 Sherwood V, Recino A, Jeffries A, Ward A, Chalmers AD: The N-terminal RASSF family: a new group of Ras-association-domain-containing proteins, with emerging links to cancer formation. Biochem J 2010;425:303-311.

11 Dansranjavin T, Wagenlehner F, Gattenloehner S, Steger K, Weidner W, Dammann R, Schagdarsurengin U: Epigenetic down regulation of RASSF10 and its possible clinical implication in prostate carcinoma. Prostate 2012;72:1550-1558.

12 Helmbold P, Richter AM, Walesch S, Skorokhod A, Marsch W, Enk A, Dammann RH: RASSF10 promoter hypermethylation is frequent in malignant melanoma of the skin but uncommon in nevus cell nevi. J Invest Dermatol 2012;132:687-694.

13 Hill VK, Underhill-Day N, Krex D, Robel K, Sangan CB, Summersgill HR, Morris M, Gentle D, Chalmers AD, Maher ER, Latif F: Epigenetic inactivation of the RASSF10 candidate tumor suppressor gene is a frequent and an early event in gliomagenesis. Oncogene 2011;30:978-989.

14 Xue WJ, Feng Y, Wang F, Li P, Liu YF, Guo YB, Wang ZW, Mao QS: The value of serum RASSF10 hypermethylation as a diagnostic and prognostic tool for gastric cancer. Tumour Biol 2016;37:1124911257.

15 Guo J, Yang Y, Yang Y, Linghu E, Zhan Q, Brock MV, Herman JG, Zhang B, Guo M: RASSF10 suppresses colorectal cancer growth by activating P53 signaling and sensitizes colorectal cancer cell to docetaxel. Oncotarget 2015;6:4202-4213.

16 Jin Y, Cao B, Zhang M, Zhan Q Herman JG, Yu M, Guo M: RASSF10 suppresses hepatocellular carcinoma growth by activating P53 signaling and methylation of RASSF10 is a docetaxel resistant marker. Genes Cancer 2015;6:231-240.

17 Liu M, Li C, Pazgier M, Li C, Mao Y, Lv Y, Gu B, Wei G, Yuan W, Zhan C, Lu WY, Lu W: D-peptide inhibitors of the p53-MDM2 interaction for targeted molecular therapy of malignant neoplasms. Proc Natl Acad Sci U S A 2010;107:14321-14326.

18 Nag S, Qin J, Srivenugopal KS, Wang M, Zhang R: The MDM2-p53 pathway revisited. J Biomed Res 2013;27:254-271.

19 Ou X, Lu Y, Liao L, Li D, Liu L, Liu H, Xu H: Nitidine chloride induces apoptosis in human hepatocellular carcinoma cells through a pathway involving p53, p21, Bax and Bcl-2. Oncol Rep 2015;33:1264-1274.

20 Morita N, Ikeda Y, Takami H: Clinical significance of p53 protein expression in papillary thyroid carcinoma. World J Surg 2008;32:2617-2622.

21 Horie S, Maeta H, Endo K, Ueta T, Takashima K, Terada T: Overexpression of p53 protein and MDM2 in papillary carcinomas of the thyroid: Correlations with clinicopathologic features. Pathol Int 2001;51:11-15.

22 Zou M, Baitei EY, Al-Rijjal RA, Parhar RS, Al-Mohanna FA, Kimura S, Pritchard C, Binessa HA, Alzahrani AS, Al-Khalaf HH, Hawwari A, Akhtar M, Assiri AM, Meyer BF, Shi Y: TSH overcomes Braf(V600E)-induced senescence to promote tumor progression via downregulation of p53 expression in papillary thyroid cancer. Oncogene 2016;35:1909-1918.

23 Liu M, Li C, Pazgier M, Mao Y, Lv Y, Gu B, Wei G, Yuan W, Zhan C, Lu WY, Lu W: D-peptide inhibitors of the p53-MDM2 interaction for targeted molecular therapy of malignant neoplasms. Proc Natl Acad Sci U S A 2010;107:14321-14326.

-24 Carvalheira G, Nozima BH, Cerutti JM: microRNA-106b-mediated down-regulation of C1orf24 expression induces apoptosis and suppresses invasion of thyroid cancer. Oncotarget 2015;6:28357-28370.

25 Li Z, Chang X, Dai D, Deng P, Sun Q: RASSF10 is an epigenetically silenced tumor suppressor in gastric cancer. Oncol Rep 2014;31:1661-1668.

-26 Wei Z, Chen X, Chen J, Wang W, Xu X, Cai Q: RASSF10 is epigenetically silenced and functions as a tumor suppressor in gastric cancer. Biochem Biophys Res Commun 2013;432:632-637.

27 Yang GC, Stern CM, Messina AV: Cystic papillary thyroid carcinoma in fine needle aspiration may represent a subset of the encapsulated variant in WHO classification. Diagn Cytopathol 2010;38:721-726.

28 Cerutti JM, Delcelo R, Amadei MJ, Nakabashi C, Maciel RM, Peterson B, Shoemaker J, Riggins GJ: A preoperative diagnostic test that distinguishes benign from malignant thyroid carcinoma based on gene expression. J Clin Invest 2004;113:1234-1242. 


\section{Cellular Physiology Cell Physiol Biochem 2017;41:1229-1239 \begin{tabular}{l|l|l} 
DOI: 10.1159/000464386 & O 2017 The Author(s). Published by S. Karger AG, Basel \\
www.karger.com/cpb
\end{tabular}

29 Cerutti JM, Latini FR, Nakabashi C, Delcelo R, Andrade VP, Amadei MJ, Maciel RM, Hojaij FC, Hollis D, Shoemaker J, Riggins GJ: Diagnosis of suspicious thyroid nodules using four protein biomarkers. Clin Cancer Res 2006;12:3311-3318.

30 Carvalheira GM, Nozima BH, Riggins GJ, Cerutti JM: DDIT3, STT3A (ITM1), ARG2 and FAM129A (Niban, C1orf24) in diagnosing thyroid carcinoma: variables that may affect the performance of this antibodybased test and promise. Mod Pathol 2013;26:611-613.

-31 Zhao S, Wang Q, Li Z, Ma X, Wu L, Ji H, Qin G: LDOC1 inhibits proliferation and promotes apoptosis by repressing NF-kappaB activation in papillary thyroid carcinoma. J Exp Clin Cancer Res 2015;34:146.

-32 Lee CK, Lee JH, Lee MG, Jeong SI, Ha TK, Kang MJ, Ryu BK, Hwangbo Y, Shim JJ, Jang JY, Lee KY, Kim HJ, Chi SG: Epigenetic inactivation of the NORE1 gene correlates with malignant progression of colorectal tumors. BMC Cancer 2010;10:577.

33 Pfeifer GP, Dammann R: Methylation of the tumor suppressor gene RASSF1A in human tumors. Biochemistry (Mosc) 2005;70:576-583.

34 Wen Y, Wang Q, Zhou C, Yan D, Qiu G, Yang C, Tang H, Peng Z: Decreased expression of RASSF6 is a novel independent prognostic marker of a worse outcome in gastric cancer patients after curative surgery. Ann Surg Oncol 2011;18:3858-3867.

35 Peng H, Liu H, Zhao S, Wu J, Fan J, Liao J: Silencing of RASSF3 by DNA hypermethylation is associated with tumorigenesis in somatotroph adenomas. PLoS One 2013;8:e59024.

-36 Richter AM, Walesch SK, Wurl P, Taubert H, Dammann RH: The tumor suppressor RASSF10 is upregulated upon contact inhibition and frequently epigenetically silenced in cancer. Oncogenesis 2012;1:e18.

-37 van der Weyden L, Adams DJ: The Ras-association domain family (RASSF) members and their role in human tumourigenesis. Biochim Biophys Acta 2007;1776:58-85.

-38 Hanahan D, Weinberg RA: The hallmarks of cancer. Cell 2000;100:57-70.

-39 Del Re DP, Matsuda T, Zhai P, Gao S, Clark GJ, Van Der Weyden L, Sadoshima J: Proapoptotic Rassf1A/ Mst1 signaling in cardiac fibroblasts is protective against pressure overload in mice. J Clin Invest 2010;120:3555-3567.

40 Oceandy D, Pickard A, Prehar S, Zi M, Mohamed TM, Stanley PJ, Baudoin-Stanley F, Nadif R, Tommasi S, Pfeifer GP, Armesilla AL, Cartwright EJ, Neyses L: Tumor suppressor Ras-association domain family 1 isoform A is a novel regulator of cardiac hypertrophy. Circulation 2009;120:607-616.

-41 Schagdarsurengin U, Richter AM, Wohler C, Dammann RH: Frequent epigenetic inactivation of RASSF10 in thyroid cancer. Epigenetics 2009;4:571-576.

42 Richter AM, Haag T, Walesch S, Herrmann-Trost P, Marsch WC, Kutzner H, Helmbold P, Dammann RH: Aberrant Promoter Hypermethylation of RASSF Family Members in Merkel Cell Carcinoma. Cancers (Basel) 2013;5:1566-1576.

43 Richter AM, Zimmermann T, Haag T, Walesch SK, Dammann RH: Promoter methylation status of Rasassociation domain family members in pheochromocytoma. Front Endocrinol (Lausanne) 2015;6:21.

44 Wang Y, Ma T, Bi J, Song B, Zhou Y, Zhang C, Gao M: RASSF10 is epigenetically inactivated and induces apoptosis in lung cancer cell lines. Biomed Pharmacother 2014;68:321-326.

$\$ 4$ Guo J, Yang Y, Linghu E, Zhan Q, Brock MV, Herman JG, Zhang B, Guo M: RASSF10 suppresses colorectal cancer growth by activating P53 signaling and sensitizes colorectal cancer cell to docetaxel. Oncotarget 2015;6:4202-4213.

46 Mirzayans R, Andrais B, Scott A, Murray D: New insights into p53 signaling and cancer cell response to DNA damage: implications for cancer therapy. J Biomed Biotechnol 2012;2012:170325. 